\title{
VERDAD SOBRE LA NO EXISTENCIA: UN PROBLEMA PARA LA TEORÍA REDUCCIONISTA DE TIM CRANE. NOTA CRÍTICA SOBRE THE OBJECTS OF THOUGHT
}

Tim Crane, The Objects of Thought, Oxford University Press, Oxford, 2013, 208 pp.

\author{
Manuel Almagro Holgado \\ Universidad de Granada \\ malmagro@correo.ugr.es
}

\section{Introducción}

El objeto de esta nota crítica es comentar algunas de las ideas que aparecen en el libro The Objects of Thought (2013) de Tim Crane y, posteriormente, discutir con más detalle una de ellas, concretamente la propuesta de Crane para explicar la verdad sobre lo que decimos acerca de cosas que no existen - en lo que sigue me referiré a esto como "problema de la no existencia"-. La tarea de explicar estos casos - en los que pensamos o decimos que es verdad que un objeto no existente tiene tal y cual propiedad - ha ocupado un lugar destacado entre los problemas filosóficos desde la antigüedad (cfr. Teeteto 189a). Así que, además de tener presencia histórica en la literatura, este problema sobresale entre las principales cuestiones filosóficas porque supone un obstáculo importante para las posiciones que parten de la concepción tradicional de lo mental y que tratan de dar cuenta de la función y naturaleza de la mente, como es el caso de Crane en la obra que comentamos aquí. ${ }^{1}$ En su libro, Crane ofrece una solución reduccionista al problema de la no existencia. ${ }^{2} \mathrm{Su}$ propuesta consiste en explicar la verdad de lo que decimos acerca de algo que no existe apelando a su dependencia de otras verdades sobre

\footnotetext{
${ }^{1}$ Nos referimos a las posiciones deudoras y continuadoras de la concepción de Franz Brentano.

2 "Una explicación reduccionista de una verdad es aquella que explica por qué tal verdad lo es apelando a otras verdades" (Crane 2013, p. 125; la traducción de las citas es mía).
} 
lo que decimos acerca de cosas que sí existen (Crane 2013, p. 137). ${ }^{3}$ Concretamente, a lo que Crane apela para explicar la verdad de lo que decimos acerca de un objeto no existente es a la representación del objeto en cuestión. Según Crane, las representaciones también forman parte de la realidad y por ello afirma el autor que la verdad de lo que predicamos sobre objetos no existentes puede explicarse o reducirse a otras verdades sobre cosas que sí existen. La idea de la propuesta reduccionista de Crane es que es verdad que un objeto no existente tiene tal y cual propiedad porque dicha propiedad depende de una representación que forma parte de la realidad. Esta tesis reduccionista se capta rápidamente con un ejemplo: la verdad de que Pegaso sea un caballo mitológico alado depende de la verdad de que hay un mito en el que Pegaso es representado como un caballo alado, o se reduce a ella. En otras palabras: es verdad que Pegaso es un caballo mitológico alado porque es verdad que existe un mito en el que Pegaso es representado de este modo. Hay un hecho contrastable empíricamente - en el ejemplo, la existencia de un mito en el que Pegaso es representado como un caballo mitológico alado- sobre el que descansa la verdad de los casos que constituyen el problema de la no existencia — en el ejemplo, la verdad de que Pegaso sea un caballo mitológico alado-. De un modo más riguroso, la tesis reduccionista de Crane puede enunciarse como sigue: la verdad o la falsedad de las proposiciones expresadas por oraciones que contienen ingredientes carentes de referencia, como el término "Pegaso" en la oración "Pegaso es un caballo mitológico alado", descansa en la verdad o la falsedad de las proposiciones expresadas por oraciones relativas a hechos del mundo $-o$ puede reducirse a esa verdad o falsedad-, concretamente aquellas oraciones que hablan de las representaciones sobre las que se apoyan las propiedades atribuidas a estos objetos que no existen, como la oración "En el mito, Pegaso es representado como un caballo mitológico alado". Éste es uno de los argumentos a los que prestaremos especial atención en esta nota

\footnotetext{
${ }^{3}$ Una de las primeras formulaciones de esta tesis que aparecen en el libro de Crane reza así: "las verdades sobre objetos no existentes deben ser explicadas en términos de verdades sobre pensamientos y otras representaciones" (p. 27). Esto no debe confundirnos. Más adelante, mientras explica la verdad de que Sherlock Holmes sea más famoso que sir Ian Blair, Crane dice: "Para que estas cosas sean verdad, todo lo que necesitamos que exista son las historias de Holmes, las representaciones de Holmes que se hace la gente, y sir Ian Blair" (p. 135). Por lo tanto, para Crane, las representaciones mentales de la gente y los sucesos psicológicos son cosas que existen, y por ello hemos enunciado la tesis utilizando la formulación final recogida en la página 137.
} 
crítica. Lo que argumentaremos aquí es que la explicación que Crane ofrece al problema de la no existencia no funciona adecuadamente en los casos en los que el predicado atribuido a un objeto que no existe tiene un uso evaluativo.

\section{Propuesta de Crane}

Una teoría que pretenda ofrecer una explicación completa sobre el funcionamiento del pensamiento debe poder explicar cómo es posible que podamos pensar en cosas que no existen - cosas tales como Superman, Papá Noel o Pegaso-. Y si, como en el caso de la propuesta de Crane, dicha teoría pretende preservar la idea de que las cosas en las que pensamos y que no existen son, en algún sentido, objetos (p. 3), entonces la teoría debe poder ofrecer algún tipo de respuesta a las preguntas (i), (ii) y (iii) siguientes:

(i) ¿Qué son los objetos no existentes?

(ii) ¿Qué es lo que permite que podamos predicar verdad o falsedad de lo que decimos acerca de cosas que no existen?

(iii) ¿Cuál es la naturaleza de la relación entre los objetos no existentes y el pensamiento?

Lidiar con estas cuestiones, tratando de resolverlas, es el objetivo de este libro de Crane. Como veremos, la respuesta que se ofrezca a (i) limita el tipo de respuesta disponible para (iii), y esto revierte en el modo de abordar (ii). Es decir, si por ejemplo se defiende que los objetos no existentes son entidades reales, entonces la relación entre un objeto no existente y el sujeto que piensa en dicho objeto debe ser una relación entre dos cosas existentes: el sujeto y el objeto. Y si éste fuera el caso, entonces uno podría explicar la verdad de que Juan piensa en Pegaso en virtud de que estas dos entidades - Juan y Pegaso - se encuentran en una determinada relación. En este sentido afirmamos que la respuesta que se ofrezca a la pregunta (i) qué son los objetos no existentes limita el tipo de respuesta disponible para la pregunta (iii) cuál es la naturaleza de la relación entre los objetos no existentes y el pensamiento, y ambas respuestas guían el camino para responder a la pregunta (ii) qué es lo que nos permite decir que es verdad que un objeto no existente tenga tal y cual propiedad. Seguiremos este mismo orden de preguntas para presentar las tesis que Crane defiende en el libro a modo de explicación de estos problemas. 
Con respecto a (i) y de acuerdo con Crane, los objetos del pensamiento son simplemente aquellas cosas en las que pensamos. Aquello en lo que pensamos puede existir o no. Cuando pensamos en un objeto que no existe, por ejemplo, en Pegaso, el objeto de nuestro pensamiento no es más que aquello en lo que pensamos, a saber, Pegaso. Que algo sea un objeto intencional — un objeto hacia el que dirigimos nuestro pensamiento - no implica necesariamente que dicho objeto exista. Ciertamente, dicho objeto puede existir, pero esto no es un requisito necesario para que algo entre a formar parte del conjunto de cosas que pueden ser objetos intencionales. Si pensamos en un cómico español contemporáneo, por ejemplo, en Ignatius Farray, entonces el objeto intencional de nuestro pensamiento sí es una entidad existente, concretamente Ignatius Farray. Sin embargo, si pensamos en Pegaso, el objeto de nuestro pensamiento no es ninguna entidad existente; es simplemente Pegaso. Que algo sea un objeto intencional no implica que dicho objeto sea una entidad existente en parte debido a, según Crane, la cantidad de tipos de cosas que pueden ser objetos de nuestros pensamientos - por ejemplo, cosas mentales o materiales, concretas o abstractas, existentes o no existentes, entre otras-. Para defender esto, en el capítulo 2, Crane rechaza la concepción tradicional quineana de la cuantificación; ${ }^{4}$ mantiene, en cambio, que las expresiones del lenguaje natural que sirven para cuantificar cosas - expresiones tales como "hay", "algunos", "muchos", entre otrasno conllevan compromisos ontológicos de existencia de aquello que se cuantifica. Podemos decir con sentido cosas como "algunos personajes de la Biblia no existieron", y si no hay problema en agrupar un conjunto de cosas que no existen, entonces no debe haber problema en que un pensamiento sea sobre algo que no existe. Del mismo modo que lo definitorio de la cuantificación es su función de seleccionar un conjunto de cosas, existan o no, la característica del pensamiento es estar dirigido hacia algo, exista o no. Este movimiento permite afirmar, por ejemplo, que pueden cuantificarse representaciones mentales - y que es posible explicar la relación entre el pensamiento y su objeto mediante dichas representaciones mentales-, y de esta manera el autor pone las primeras piedras sobre las que se erige su postura de lo mental.

El modo en que Crane responde a (i) descarta la posibilidad de adoptar una postura relacional ante (iii): si los objetos intencionales

\footnotetext{
${ }^{4}$ La concepción que defiende que bajo el dominio de la cuantificación sólo pueden caer cosas existentes, y que, por lo tanto, oraciones como "hay un $x$ que no existe" expresan una contradicción.
} 
no son necesariamente entidades existentes, entonces el pensamiento no es necesariamente una relación entre dos cosas existentes. Para defender que el pensamiento es relacional y que los objetos intencionales no implican necesariamente su existencia hace falta especificar qué tipo de relación particular es el pensamiento. Con este fin introduce Crane la distinción metafísica entre relación sustancial, como la referencia, y relación no sustancial (p. 9). Una relación sustancial es aquella que exige que cada una de las partes en relación exista. La referencia es un ejemplo de este tipo de relación: conecta una palabra, por ejemplo "Ana", con un objeto, Ana, y ambas cosas existen. Una relación no sustancial, por el contrario, no exige que cada una de las partes en relación exista. La intencionalidad - la relación entre el pensamiento y su objeto- es un ejemplo de este tipo de relación, afirma Crane: conecta a un sujeto con una cosa - exista o no- mediante la representación de esta última. La intencionalidad es la mera representación de algo en palabras o en el pensamiento (pp. 9 y 68), y por ello podemos pensar en cosas que no existen.

Una respuesta completa a (iii) —el problema filosófico particular de explicar cómo se relaciona el pensamiento con aquello sobre lo que el pensamiento trata - requiere una respuesta satisfactoria a (ii): el problema de la no existencia (p. 5). Esta conexión entre ambas cuestiones no es de extrañar. Si partimos de que aquello en lo que pensamos es, de algún modo, un objeto, entonces que sea verdad que Pegaso es un caballo mitológico alado y que podamos pensar con naturalidad en un objeto que no existe como Pegaso produce, en un sentido al menos, un estupor similar. Resulta igual de extraño decir que cuando pensamos estamos en una relación con un objeto que no existe, que decir que es verdad que un objeto que no existe tiene tal y cual propiedad. Según Crane, debemos explicar qué es lo que nos permite decir, por ejemplo, que nuestra creencia de que Pegaso es un caballo mitológico alado es verdadera, si queremos explicar correctamente cómo se relaciona el pensamiento con su objeto. Así, para responder a (ii) dentro del esquema reconstruido hasta el momento, Crane introduce una distinción entre dos tipos de propiedades (pp. 64-68), distinción similar a la mencionada antes entre dos tipos de relaciones. Tenemos, por un lado, propiedades sustanciales y, por otro lado, propiedades no sustanciales o pleonásticas. ${ }^{5}$ Las propiedades sustanciales son aquellas que sólo pueden ser instanciadas por entidades existentes (p. 70). Por ejemplo, la pro-

\footnotetext{
${ }^{5}$ Término acuñado por Schiffer (2003) para referir al tipo de entidad que son las proposiciones y las propiedades.
} 
piedad de ser un caballo es una propiedad sustancial: para que sea verdad que $x$ es un caballo, $x$ debe existir. Las propiedades pleonásticas, sin embargo, no conllevan compromisos de existencia, y en consecuencia pueden ser instanciadas tanto por objetos existentes como por objetos no existentes. Por ejemplo, la propiedad de ser un caballo mitológico alado es una propiedad pleonástica: para que sea verdad que $x$ es un caballo mitológico alado, $x$ no debe existir. Crane acentúa la diferencia entre estos dos tipos de propiedades en términos de su dependencia con respecto a la existencia de representaciones (representation-dependence). ${ }^{6}$ Las propiedades pleonásticas son dependientes de la representación y esto significa que un objeto $x$ sólo puede tener la propiedad pleonástica $P$ si ha sido representado de tal modo en algún lugar o por alguien alguna vez. Así, los objetos existentes pueden tener tanto propiedades sustanciales como pleonásticas. Los objetos no existentes, por el contrario, solo pueden tener propiedades pleonásticas.

Puestos estos elementos sobre la mesa ya puede comprenderse mejor cómo funciona la tesis reduccionista de Crane que mencionábamos al principio: decimos que es verdad que Pegaso tiene la propiedad pleonástica de ser un caballo mitológico alado porque hay un mito en el que se representa a Pegaso con esta propiedad pleonástica. Las representaciones de las que estas propiedades dependen forman parte de la realidad (p. 120). Éste es el modo en el que el autor explica la verdad de la proposición expresada por una oración del tipo "Pegaso es un caballo mitológico alado".

\section{Límites de la tesis reduccionista}

Como apuntamos al principio, parte del objetivo de esta nota crítica es discutir la tesis reduccionista que Crane plantea como solución al problema de la no existencia recogido en (ii). En el capítulo 6 de su libro, el propio Crane señala y responde a una objeción que podría hacérsele a su modo de explicar la verdad predicada de lo que decimos acerca de cosas que no existen. Aunque la crítica que Crane comenta es distinta de la que queremos discutir nosotros, merece la pena presentarla antes de pasar a la discusión que nos interesa. La objeción en cuestión es la siguiente: si, por ejemplo, Ana y Cristina

${ }^{6}$ Crane utiliza el término "representation-dependent properties" en el sentido en que lo utilizó McGinn (2002): propiedades que dependen de que el objeto que las instancia haya sido representado de un modo particular en el pensamiento, en el lenguaje, etcétera. 
están pensando en Pegaso, parece que ambas personas están pensando en un mismo objeto particular no existente. Pero si los objetos no existentes no son entidades y las propiedades que les atribuimos a este tipo de objetos son propiedades pleonásticas dependientes de la representación, ¿en qué sentido decimos que Ana y Cristina están pensando en el mismo objeto? En otras palabras, si aceptamos que los objetos no existentes no son entidades y decimos que es verdad que tienen determinadas propiedades en virtud de su dependencia de lo que existe, a saber, de las representaciones que tenemos de estos objetos, entonces ¿en qué medida esta posición - la de Crane- explica nuestra intuición de que hay cierta identidad entre aquello en lo que piensa Ana y aquello en lo que piensa Cristina? Crane responde a esta posible objeción del siguiente modo: por un lado, mantiene que la identidad puede predicarse exclusivamente de objetos existentes. En el caso de los objetos no existentes, la única identidad que tenemos es identidad intencional (Geach 1967), esto es, similitudes en las representaciones o archivos mentales (mental files) que los agentes tienen acerca de los objetos no existentes en cuestión. Por otro lado, Crane afirma que podemos tener pensamientos específicos y singulares sobre objetos que no existen. Cuando nos formamos un pensamiento singular, que es un pensamiento específico sobre un objeto particular, tratamos de referir a algún objeto. Pero la referencia puede fallar. Cuando éste es el caso, tenemos un pensamiento singular sobre un objeto que no existe. De este modo, de la argumentación de Crane se desprende que cuando Ana y Cristina piensan en Pegaso, Ana y Cristina tienen un pensamiento singular distinto sobre el mismo objeto no existente, y comparten cierta similitud en sus respectivas representaciones mentales acerca del objeto de su pensamiento: Pegaso.

Así es como Crane se deshace de esta objeción que él mismo plantea: apela a la función psicológica que cumplen los pensamientos singulares y a las similitudes de las representaciones mentales relativas al objeto intencional no existente para explicar cómo dos personas que piensan en Pegaso están pensando en el mismo objeto. En lo que sigue discutiremos más profundamente otra dificultad que exhibe el modo en el que Crane se enfrenta al problema de la no existencia y que no aparece recogida en el libro. Para ello, presentaremos la explicación que el propio autor ofrece de cuatro casos particulares de este problema (pp. 134-136) y los utilizaremos para tratar de exponer con la mayor claridad posible la dificultad mencionada. Los casos son los siguientes: 
1) Vulcano fue un planeta postulado por Le Verrier en 1859 para explicar las perturbaciones en la órbita de Mercurio. Vulcano fue postulado usando los mismos métodos que Le Verrier había usado para introducir Neptuno en 1846.

2) Sherlock Holmes es más famoso que cualquier detective vivo; por ejemplo, Sherlock Holmes es más famoso que sir Ian Blair.

3) Pegaso es un caballo mitológico alado; en el mito, Pegaso cobró vida de la sangre de Medusa, la gorgona que mató Perseo.

4) Siegfried es uno de los personajes menos atractivos de todas las obras dramáticas.

La explicación que ofrece Crane de estos casos es la siguiente: afirmar la verdad de (1) es afirmar que Vulcano tiene la propiedad pleonástica dependiente de la representación de ser postulado por Le Verrier en 1859, y decir esto es asumir que Le Verrier se representó a Vulcano como un planeta. En otras palabras, el hecho de que Le Verrier creyera que Vulcano era un planeta $-o$ que se lo representara como un planeta - es suficiente para explicar que podamos decir que es verdad que Vulcano tiene la propiedad pleonástica de ser un planeta postulado. En el ejemplo (2), la verdad de que Sherlock Holmes esté en la relación no sustancial ser más famoso que con sir Ian Blair depende del hecho de que haya más cantidad de gente que haya oído hablar de las historias de Sherlock Holmes — se lo haya representado - que de Ian Blair. En (3), podemos afirmar la verdad de que Pegaso tiene la propiedad pleonástica de ser un caballo mitológico alado o de haber cobrado vida de la sangre de Medusa porque existe un mito en el que Pegaso es representado de tal modo. Por último, en el ejemplo (4), lo que explica la verdad de que Siegfried tenga la propiedad pleonástica de ser un personaje poco atractivo es un conjunto de hechos de la obra dramática donde Siegfried es representado, concretamente las partes donde es representado como violento, tozudo, desconsiderado, ignorante y descuidado. ${ }^{7}$

${ }^{7}$ Hay una diferencia entre las afirmaciones acerca de objetos no existentes relativos a una teoría (como la afirmación (l) sobre Vulcano) y las afirmaciones acerca de objetos no existentes relativos a mitos o ficciones (como las otras tres afirmaciones). La diferencia tiene que ver con que en una teoría hay "operadores implícitos" funcionando que no necesariamente hay en un mito o ficción. La postulación de un objeto teórico debe ser consistente con los estándares de la teoría misma, mientras que en un mito o en una ficción los estándares no tienen por qué ser exclusivos del mito o la ficción; pueden ser estándares externos a ellos. 
En los ejemplos (1), (2) y (3), si el hecho en el que descansa la verdad de cada una de estas afirmaciones es el caso, entonces parece que necesariamente las proposiciones que expresan estas oraciones deben ser verdaderas. No tiene sentido decir que es el caso que más cantidad de gente ha oído hablar de Sherlock Holmes que de Ian Blair y seguidamente negar que Sherlock Holmes sea más famoso que Ian Blair, o afirmar que existe un mito en el que Pegaso es representado como un caballo alado y rechazar que Pegaso sea un caballo mitológico alado. En el caso (4), sin embargo, no necesariamente ocurre lo mismo. Que sea el caso que Siegfried es representado en una obra dramática como violento, tozudo, desconsiderado, ignorante y descuidado no implica necesariamente que sea verdad que Siegfried es uno de los personajes menos atractivos de todas las obras dramáticas; a pesar de que Siegfried esté representado de este modo, Siegfried podría resultar un personaje atractivo para alguien. Crane parece ser consciente de este problema: "Describirlo [a Siegfried] como poco atractivo es un juicio basado en los hechos que las historias cuentan. Por supuesto, esto puede discutirse. Pero en la discusión, los únicos hechos a los que necesitamos apelar son los hechos de la obra misma" (p. 136). Sin embargo, por el tipo de respuesta que Crane ofrece aquí, podemos concluir que al menos no es completamente consciente del problema. El autor tiene razón y su estrategia queda a salvo si con (4) lo que queremos decir es algo parecido a que, después de hacer un muestreo, la mayoría de personas encuestadas ha seleccionado a Siegfried como el personaje menos atractivo de todas las obras dramáticas por ser violento, tozudo, desconsiderado, ignorante y descuidado, o por otros hechos relatados en la obra en cuestión. Si interpretamos (4) así, entonces esta afirmación podría analizarse del mismo modo que las tres afirmaciones anteriores y Crane tendría razón. No tiene sentido decir, interpretando (4) como lo acabamos de hacer, que es el caso que la mayoría de personas - quitemos lo de "personas encuestadas" para darle más fuerza al ejemplo - han seleccionado a Siegfried como el personaje menos atractivo de las obras dramáticas y seguidamente negar que Siegfried es uno de los personajes menos atractivo de todas las obras dramáticas.

Ahora bien, si con (4) lo que hacemos es una evaluación personal, entonces la estrategia de reducir la verdad de (4) a la verdad de un conjunto de descripciones no funciona. Éste podría ser, digamos, el punto de vista de uno de los encuestados que no ha seleccionado a Siegfried como el personaje menos atractivo de las obras dramáticas. Alguien podría decir: es verdad que Siegfried es un personaje violen- 
to, tozudo, etc., pero a mí me parece muy atractivo. Incluso si a esta persona se le preguntara por qué le parece tan atractivo Siegfried, tiene sentido imaginar que el ficticio fan de Siegfried ofreciera razones que no tuvieran nada que ver con los hechos relatados en la obra misma.

Hay al menos dos razones para defender que, bajo determinada interpretación o determinado uso, la verdad de (4) no pueda reducirse a la verdad de un conjunto cerrado de descripciones.

La primera razón es la distinción entre adjetivos unidimensionales y multidimensionales (McNally y Stojanovic 2014). Los adjetivos unidimensionales tales como "largo" o "famoso" se caracterizan por aplicarse bajo un único criterio: la longitud o la cantidad de personas que han oído hablar de algo. Los adjetivos multidimensionales, por el contrario, se definen por tener más de un criterio de aplicación. Por ejemplo, un entorno rural puede ser bonito o atractivo en virtud del tipo de vegetación que tiene, del contraste que hay entre los distintos colores que posee, de la disposición que presentan los elementos que lo componen, de lo que permite experimentar, etc. En este sentido, el adjetivo "bonito" sería multidimensional. La idea es que tanto las dimensiones de las que dependen los adjetivos multidimensionales, como el peso relativo de las mismas, pueden variar de contexto a contexto, mientras que esto no ocurre necesariamente con los adjetivos denominados unidimensionales. El adjetivo "atractivo" que aparece en (4) es un adjetivo multidimensional, y esto significa que a pesar de que dos hablantes coincidan en decir de algo que es atractivo, cada uno de ellos puede estar aludiendo a una dimensión distinta, y por lo tanto no necesariamente la verdad de (4) descansará sobre un conjunto cerrado de descripciones previamente señalado. Esto sólo supone un problema para la explicación concreta ofrecida por Crane de la verdad de (4): aunque Siegfried sea representado como violento, tozudo, etc., alguien podría aludir a otros hechos relatados en la obra al afirmar que Siegfried es poco atractivo. Crane advierte esta posible crítica, y por eso afirma que a pesar de que puede ser discutido cuáles son los hechos sobre los que descansa la verdad de (4), en la discusión sólo necesitamos apelar a los hechos recogidos en la obra en la que aparece Siegfried representado. De este modo, la estrategia reduccionista de Crane sigue funcionando: aunque no podemos dar previamente el conjunto de hechos sobre los que descansa la verdad de (4), podemos explicar la verdad de (4) en virtud de la verdad de un conjunto de hechos representados en la obra.

La segunda razón, sin embargo, sí compromete la estrategia general de Crane para explicar la verdad de lo que decimos acerca de cosas 
que no existen, y es la siguiente: el significado de un término, un predicado o una oración, en su uso evaluativo, no puede reducirse al significado de un conjunto de oraciones usadas descriptivamente. Hay distintas formas de explicar este fenómeno. La falacia naturalista de Hume (1739-1740) o el argumento de la pregunta abierta de Moore (1903/1988) inciden en esta idea de que lo normativo y lo evaluativo no pueden reducirse a lo descriptivo. En lo que sigue trataremos de explicar esta idea apoyándonos en observaciones más contemporáneas que las ofrecidas por Hume y Moore. La idea es que un término como "atractivo" puede usarse en dos sentidos bien distintos: cuando decimos de algo que es atractivo, podemos querer decir que ese algo es atractivo con respecto a un estándar particular - y en este caso lo que haríamos es decir que tal cosa tiene unas determinadas características en función de la dimensión a la que apelemos-, o simplemente querer decir que ese algo nos parece atractivo, y no necesariamente porque tenga un conjunto particular de características. Ésta es la consideración en la que vamos a hacer hincapié aquí para tratar de explicar este fenómeno: la distinción entre dos tipos de usos de un término, predicado u oración. ${ }^{8}$ La diferencia que advirtió Wittgenstein entre el uso trivial o relativo y el uso ético o absoluto de un predicado como "es bueno" (cfr. Wittgenstein 1965/2011, p. 35) puede ayudarnos a introducir esta distinción. Podemos afirmar cosas como que este libro es bueno o que tal camino es bueno queriendo decir con ello que este libro cumple ciertos estándares de calidad o que tal camino es el más estable y corto para llegar a un destino concreto. Éstos serían ejemplos de usos relativos del predicado "es bueno", y se caracterizan por ser descripciones de estados de cosas. En palabras de Wittgenstein: "cada juicio de valor relativo es un mero enunciado de hechos" (1965/2011, pp. 35-36). Con estas afirmaciones simplemente estamos hablando acerca de cómo son las cosas. Pero

${ }^{8}$ La crítica que planteamos aquí es ortogonal a la distinción entre objetos no existentes relativos a teorías y objetos no existentes relativos a mitos y ficciones literarias. Es cierto que habitualmente evaluamos un objeto no existente relativo a una teoría en virtud de los estándares de las teorías en cuestión, mientras que los objetos no existentes relativos a mitos y ficciones literarias suelen ser evaluados con respecto a estándares externos. En este sentido, alguien podría defender que la crítica es más pertinente para los casos de objetos no existentes relativos a mitos y ficciones literarias. No obstante, la crítica abarca ambos casos: alguien podría hacer una evaluación sobre un objeto no existente relativo a una teoría y que la evaluación tuviera que ver con lo que el sujeto ha experimentado estudiándolo (utilizando un estándar externo a la teoría), y alguien podría evaluar un objeto no existente relativo a un mito o a una ficción literaria en virtud de cuánto se ajusta ese objeto a los rasgos propios del mito o la ficción (utilizando un estándar interno al mito o ficción). 
también podemos afirmar que es bueno comportarse de tal y cual modo no queriendo decir con ello que dicho comportamiento sea bueno con respecto a unas normas o a un objetivo particular, sino afirmando que todo el mundo debería comportarse así independientemente de las leyes vigentes y de sus propósitos personales. Éste sería un ejemplo de uso absoluto del predicado "es bueno", y con este uso lo que hacemos es "ir más allá del mundo" (1965/2011, p. 43), que en el paradigma de la época tractariana del autor significa no describir estados de cosas. Cuando hacemos un uso absoluto de un predicado, lo que decimos no puede reducirse a un conjunto de hechos porque precisamente no estamos describiendo nada. Por lo tanto, una misma oración puede usarse en dos sentidos bien distintos. Wittgenstein distingue entre usos relativos y usos absolutos. Nosotros llamaremos "descriptivos" y "evaluativos" a estos dos tipos de usos: los primeros informan acerca de cómo son las cosas en el mundo, los segundos no describen nada. En términos más actuales, podemos dar cuenta de esta distinción entre diferentes tipos de usos de un predicado apelando a la siguiente formulación ofrecida por Nate Charlow: la información que transmitimos al proferir una oración puede ser de tipo locacional u orientacional (Charlow 2014, p. 640; véase también Lewis 1979). Cuando es de tipo locacional, la información que comunicamos permite localizarnos entre un conjunto de posibilidades o mundos posibles relevantes. Cuando es de tipo orientacional, la información que transmitimos, por el contrario, no permite localizarnos en el espacio lógico. De este modo, si con (4) hablamos del hecho de que un conjunto de personas encuestadas ha seleccionado a Siegfried como el personaje menos atractivo de las obras dramáticas, (4) sería de tipo locacional: la información ofrecida nos permite eliminar mundos posibles - aquellos en los que la mayoría de encuestados no ha elegido dicha opción, por ejemplo - entre un conjunto de posibilidades relevantes. Por el contrario, si con (4) lo que decimos es que Siegfried nos parece el personaje menos atractivo de todas las obras dramáticas, (4) sería de tipo orientacional: la información que comunicamos no nos permite eliminar mundos posibles entre un conjunto de posibilidades relevantes, pues no hablamos de hechos, no hablamos acerca de cómo son las cosas en el mundo, y por lo tanto con dicha información no podemos localizarnos en el espacio lógico.

Utilizando este esquema en la propuesta de Crane, su explicación de la verdad del ejemplo (4) sólo funciona cuando usamos (4) en sentido relativo - en términos de Wittgenstein - o cuando la información comunicada es de tipo locacional —en términos de Charlow-. En 
cambio, cuando usamos (4) en sentido absoluto o cuando lo comunicado con (4) es de tipo orientacional, la verdad de lo afirmado no puede reducirse a un conjunto de hechos o descripciones. En otras palabras: cuando usamos (4) en sentido descriptivo, podemos explicar la verdad de (4) apelando a un conjunto de hechos representados en algún lugar. Cuando usamos (4) en sentido evaluativo, no necesariamente podemos explicar la verdad de (4) apelando a un conjunto de hechos representados en algún lugar.

\section{Conclusión}

La conclusión de esta discusión es que la tesis reduccionista propuesta por Crane en el libro como solución al problema de la no existencia no funciona para los casos en los que el uso del predicado verdadero que se le atribuye a un objeto que no existe es normativo, evaluativo, absoluto o de tipo orientacional. Además, cuando el uso del predicado verdadero que se le atribuye a un objeto que no existe es descriptivo pero contiene un adjetivo multidimensional, el conjunto de hechos representados sobre el que descansa la verdad del predicado atribuido al objeto no existente en cuestión no es un conjunto cerrado, y variará de contexto a contexto. Ésta es, en resumen, una de las dificultades que presenta la propuesta de Crane para explicar la verdad de lo que decimos sobre cosas que no existen y que no aparece recogida en su obra.

Puede objetarse en contra de nuestra crítica que el problema señalado aquí no es un problema particular de la propuesta de Crane, sino más bien un rasgo que exhibe el lenguaje en general. Gran parte de las oraciones de nuestro lenguaje, si no todas, pueden utilizarse tanto en sentido descriptivo como en sentido evaluativo. Alguien puede informar, por ejemplo, de los rasgos de una persona y con ello estar haciendo una evaluación. Imaginemos a un grupo de personas discutiendo acerca de si debe representarles en televisión Juan o Pedro. $\mathrm{Si}$, por ejemplo, un simpatizante de Juan dice en tal contexto "Pedro mide un metro y medio", y no hay ningún requisito de estatura para ser el representante de dicho grupo en televisión, tal afirmación podría tener un uso evaluativo: lo que podría estar diciendo con ella sería algo parecido a "no me parece bien que nos represente alguien que mida un metro y medio" o "prefiero que nos represente alguien que no sea de este modo", y esto es una evaluación. Es cierto que tanto la posibilidad de usar una misma oración en sentido descriptivo y en sentido evaluativo como que el significado de una oración, en su uso evaluativo, no pueda reducirse a un conjunto 
de descripciones son rasgos que presenta el lenguaje en general y no implicaciones de la propuesta de Crane. No obstante, la tesis reduccionista que ofrece el autor en su libro para explicar la verdad predicada sobre lo que decimos acerca de cosas que no existen asume que se puede explicar la verdad o la falsedad de cualquier afirmación relativa a un objeto no existente en virtud de otra cosa. Según Crane, todo lo que digamos de, por ejemplo, Superman, podrá ser declarado verdadero o falso en virtud de una representación concreta. Crane consigue explicar así la verdad de que Superman suela llevar capa o tenga el pelo negro, digamos. Sin embargo, como hemos visto antes, la verdad de oraciones como "Superman es el superhéroe más valiente" o "Superman es buena persona", en su uso evaluativo, no puede reducirse al modo en que Superman es representado en las historias de DC Comics. Por consiguiente, no es cierto que pueda explicarse la verdad o la falsedad de cualquier afirmación sobre un objeto que no existe en virtud de un conjunto de hechos representados en algún lugar. Habitualmente evaluamos tanto objetos que no existen como las propiedades que les atribuimos a estos objetos, y habitualmente predicamos verdad de nuestras afirmaciones evaluativas relativas a estos objetos, como ocurre en el caso de (4). La teoría de Crane no parece dar cuenta de estos casos, y, en consecuencia, su teoría no parece ser una teoría completamente adecuada. ${ }^{9}$

Finalmente, y para cerrar esta nota crítica, cabe decir algo general acerca del libro. A pesar de la objeción que hemos planteado aquí, e independientemente de la simpatía filosófica que se tenga por una propuesta de este tipo o del costo que puedan tener algunos de los problemas que pudieran derivarse de ella, el libro de Tim Crane presenta el problema de la intencionalidad con claridad; plantea una argumentación en general sólida y natural; discute diversas cuestiones centrales para la filosofía de la mente desde una perspectiva contemporánea que cuenta con muchos seguidores; plantea argumentos y propuestas originales y verdaderamente útiles, y ofrece el estímulo necesario para seguir reflexionando sobre estas cuestiones. Por todo ello, The Objects of Thought es una obra completamente recomendable tanto para quienes desean introducirse en el problema de la

\footnotetext{
${ }^{9}$ No es el objetivo de esta nota crítica tratar de ofrecer una solución al problema que plantea la teoría de Crane, sino simplemente presentar y discutir esta objeción. No obstante, sospecho que defender que la verdad de cualquier afirmación depende de cómo es el mundo, que las representaciones son también hechos en algún sentido y que las representaciones psicológicas deben desempeñar un papel central en la explicación de lo mental va a limitar la explicación de algún modo parecido al que hemos presentado aquí.
} 
intencionalidad, como para lectores ya habituados a pensar y leer acerca de estos temas. ${ }^{10}$

\section{BIBLIOGRAFÍA}

Charlow, N., 2014, "The Problem with the Frege-Geach Problem", Philosophical Studies, vol. 167, no. 3, pp. 635-665.

Crane, T., 2013, The Objects of Thought, Oxford University Press, Oxford. Geach, P., 1967, "Identity", Review of Metaphysics, vol. 21, no. 1, pp. 312.

Lewis, D., 1979, "Attitudes De Dicto and De Se", The Philosophical Review, vol. 88, no. 4, pp. 513-543.

McGinn, C., 2002, Logical Properties, Oxford University Press, Oxford.

McNally, L., e I. Stojanovic, 2014, “Aesthetic Adjectives", en J. Young (comp.), The Semantics of Aesthetic Judgment, Oxford University Press, Oxford, pp. 17-37.

Moore, G.E., 1903 (1988), Principia Ethica, Prometheus Books, Nueva York. [Versión en castellano: Principia Ethica, 2a.ed. rev. y amp., ed. e introd. T. Baldwin, trad. A. García Díaz y A.I. Stellino, Instituto de Investigaciones Filosóficas-UNAM, México, 1997.]

Schiffer, S., 2003, The Things We Mean, Clarendon Press, Oxford.

Wittgenstein, L., 1965 (2011), Conferencia sobre ética. Con dos comentarios sobre teoría del valor, trad. Fina Birulés, Paidós, Barcelona, 1990.

Recibido el 5 de julio de 2017; revisado el 1 de diciembre de 2017; aceptado el 14 de diciembre de 2017.

${ }^{10}$ Agradezco a Daniel Galdeano y Alba Moreno sus comentarios a una versión anterior de este texto, y especialmente le doy las gracias a Neftalí Villanueva por las beneficiosas conversaciones que hemos sostenido, así como por sus observaciones, sugerencias y comentarios durante todo el desarrollo de esta nota crítica, todo lo cual sin duda ha contribuido a mejorarla. 